\title{
Stochastic Structure of Brokered Foreign Exchange Auctions
}

\author{
Ryosuke Wada \\ Department of Economics \\ Otaru University of Commerce \\ 3-5-21 Midori, Otaru, 047-8501, Japan \\ rwada@res.otaru-uc.ac.jp \\ phone:81-134-27-5319 \\ Fax:81-134-27-5213
}

October 10, 2005

\begin{abstract}
Our subject is a brokered foreign exchange auction. It is done in continuous time. FX Dealers submit orders asynchronously. Heterogeneous expectations and arrivals of retail transactions interact. They together randomly determine a sequence of transaction prices. We provide a model of order flow generation. This fills what has lacked in the existing microstructure literature. Trading volume and volatility are endogenous. We derive causes of their variability and hence, correlation between them. For example, ceteris paribus, the heterogeneous expectations increases volatility.
\end{abstract}

We abstract the market microstructure as a collection of continuous time Markov processes. We define finite number of states of the market. The auction takes forms of transitions between these states. For this argument, we construct an infinitesimal operator. This makes it possible to derive transition probabilities of the market for any time interval.

Other features are as follows: (i) Arrivals processes of buyers and sellers substitute demand and supply schedules to handle asynchronous transactions. (ii) Two sources generate the order arrivals. One is dealers' revising expectations and the other is dealers' retail transactions. (iii) The bench mark for dealer's decision making is not an expected equilibrium but the first peak or trough on the 
expected time path. (iv) Dealers take into account of a distribution of reservation prices to choose their own. (v) Dealers, having heterogeneous reservation prices, as a whole absorb excess demand from the macro fundamentals. Price changes enough to do so. (vi) We derive stochastic characteristics of bid rate. Using it, we approximate change in transaction price volatility.

Keywords: Foreign Exchange, Volume and Volatility, Heterogeneous Expectations, Continuous Time Markov Process, Infinitesimal Operator 


\section{Introduction}

\subsection{Structure underlying Signed Orders}

Auctions in many financial markets are continuous. Transactions take place at bid or ask. In such auctions, it does matter which side takes initiative. The side being hit moves backward. The signed orders move price. Transaction volume that is signed is called "order flow." Lyons[1] provides empirical observations that order flow set trends in foreign exchange rates. If the demand shift changed the price, then we need to analyze causes of the demand shift. Similarly, we need to analyze what causes changes in order flow. Lyons[1] does not investigate a structure underlying order flow. It lacks tools. Our paper fills what it lacks. Our model is about the structure which generates signed orders.

Our model is tailored to brokered auctions in foreign exchange market. Yet, our model provides an mechanism to handle an issue which are common in many financial markets. In financial markets, investors have to take other investors' view into account: He may be correct but he may be a loser if he is outnumbered. "The grater fool theory" of stock markets describes another aspect of the same issue. In our paper, foreign exchange dealers are aware of that expectations are heterogeneous among them. They try to fathom distribution of expectations. Based on this distribution, dealers form own expectations. Our FX dealers take into account each other what others are thinking. We present a model about this.

\subsection{Trading Mechanism as Stochastic Processes}

Our approach consists of novel applications of stochastic processes on microstructure. Two sources generate order flow. They are firstly dealer's expectation revising process and secondly dealer's retail transaction arrival process. The former is two state continuous-time Markov process. In one state, a given dealer is ready to have open position. And in the other, he is not. Sojourn times in these two states are exponentially distributed. Times of switches are random in continuous time. When switch occurs, he tries to adjust his position. He submits "market order." This is a fraction of order generations.

Dealer's retail transactions also generate orders. Each dealer has retail customers. Transactions with them are modeled as Poisson arrivals. Their arrivals deviate dealer's position from his chosen level. He tries to recover it. Through the position adjustments, he pass retail transactions on to inter-dealer wholesale market. Retail transactions appear Poisson arrival there. Combined individual dealer's retail transactions become again Poisson. Order flows from macro economy constitute two Poisson processes, arrivals of buyers and sellers in FX market.

We define a set of "inventory state of the market". The market goes through stochastic transitions between these inventory states. The state is identified with 
a pair of numbers. The first element is the number of dealers who are ready to have open positions. The second element is net retail transactions. Two sources generate arrivals of orders. An arrival of order causes transition between the inventory states. We construct "an infinitesimal operator" for this continuous time Markov transitions. We use the infinitesimal operator in the Kolmogorov's backward differential equation. By solving Kolmogorov's backward equation, we can obtain transition probabilities between any inventory states.

Thus far, we set up a structure which sets volume stochastically. Next we provide a structure which sorts out sign. This structure also characterizes volatility. We have two features. The first is introduction of a distribution function of reservation prices. Dealer's expectation switches between two states as explained above. When he enters one of the state, he picks up his reservation price. He abandons it when he exits. This random value follows a given distribution price. He compares his value with bid/ask in the market. The bid rate here means the maximum of existing limit order buying prices. Depending on this comparison, if he submits a market order, then a signed transaction is generated.

The second feature is approximating transaction price by bid rate. We approximate variation of transaction price volatility by that of bid rate. Transaction price change consists of two elements; one due to jumps between bid and ask and the other due to change in bid/ask average. Change in transaction price volatility can be approximated by change in the bid rate volatility. Each state of the market has corresponding parameter values for the distribution function for the bid rate. We show contributing factors' influence on the bid rate's distribution function. It is not tractable to follow each jump of bid rate. However, it is possible to approximate such jumps' volatility by variance of "expected value of bid rate which is associated with each inventory state distribution function. By doing this, we show that contributing factors' effect on the volatility.

\subsection{Significance as Economic Model}

Foreign exchange market can be interpreted as an asset market where participants seek capital gain. Like many other financial markets, the auctions are continuous. Being asset market and having continuous auctions pose two theoretical issues. Our approach proposes some answers to these issues. First, it is difficult to apply equilibrium analysis on continuous auctions. Second, no truistic bench mark variable is available for investor's optimization problem. Investors' belief influence outcome and, knowing it, they choose actions.

In continuous auctions, transactions take place asynchronously. It does so anytime when two parties agree on the price. This means that before one price clears the market through the price adjustment, a transaction takes place. The pair of traders drop out. For such a process, defining demand and supply schedules explicitly and, hence, equilibrium is not truism. For this issue, we substitute demand and supply 
by arrivals of buyers and sellers. The arrival process is stochastic. They arrive randomly with different price perspectives. We distinguish expected and realized values. The realized numbers of buyers and sellers at a given time may not match.

For the second issue, our bench mark variable is the first peak or trough on expected time path. Dealers assign their values for this variable. The bench mark values are heterogeneous. Our model provides an expectation formation mechanism which all the dealers may share but which still they come up with heterogeneous benchmark value.

\subsection{Spot FX market with a Broker}

Inter-bank FX transactions have two channels. One is through brokers, and the other without broker. We model an auction in the former channel. Price quotes go through brokers. Dealers are not obliged to keep quoting both prices. Only when they want, they submit limit orders to a broker. We assume an environment of the market as follows. There is a broker. The auction is continuous and double sided. Buyers and sellers compete in their own sides. Among submitted limit orders, the broker keeps announcing the best buying and selling prices. Spot foreign exchange is the commodity to trade. The market is geographically local. It has trading hours. There are many dealers. Let's take a representative dealer. He is risk neutral. His objective is daily profit maximization. He is allowed to have open position up to one transaction unit. Deliveries and settlements are scheduled for another day. So no interest cost incurs in order to have intra-day open position. He trades with retail customers any time they want during business hours. Their arrivals are asynchronous and random.

Analyses of continuous auction in financial market need extra approach. Firstly, transactions take place asynchronously. To handle this, we use arrival intensity of buyers and sellers, instead of demand and supply. The arrival intensity is expected number of arrivals per unit time. Our use of the arrival intensity is in line with Garman [2] and Amihud, Yakov and Mendelson [3]. The next issue poses non trivial question. At a microstructure level, distinction between equilibrium and deviation is not truism. In such a environment, adverse selection problem is not major issue. Mere perspectives change outcome. You should take advantage of errors, if many believe it. So judging dispersion of heterogeneous price perspectives becomes important to make decisions. We face the following question: How do you form price expectation rational way while you know traders' perspectives collectively influence the actual outcome? Our answer is as follows: Dealers as a whole absorb unbalanced order flow from macro fundamentals. Meanwhile, dealers submit heterogeneous limit order prices. As these limit orders, one by one, absorb the unbalanced flow, transaction prices change. Our dealer perceives this mechanism. So he tries to fathom a distribution of reservation prices among dealers. Also he tries to foresee time path of the order flow arrivals. Then he expects configuration of price's time path. Dealers may agree on such a price determination mechanism. However, still 
heterogeneous perspectives persist. It is because information is limited. The distribution of reservation prices is not observable. Also dealers know only a fraction of order flow from macro economy as their own transactions.

Organizations of the rest of this paper are as follows; In the next section, we present our model of stochastic price determination. (i) We present stochastic processes which generate order arrivals. (ii) We construct a matrix of continuous time Markov process which determines transition probabilities of the market inventory states. (iii) We define reservation price as a random variable on unit interval. We derive a distribution function of bid rates. (iv) We present a process of dealer's expectation formation. In the third section, we analyze causes of volume and volatility change and their effects. Next, conclusion and appendix follow.

\section{Stochastic Order Generations}

\subsection{Switching Process of Perspectives}

Currencies are traded 24 hours on the globe. They are done so consecutively by geographically local markets. We consider such a local market. It opens in the morning and ends in the evening. FX dealers trade in the local market. Closing hours overlaps with the opening hours of the next market. It is possible for dealers to have transaction with overseas counterparts then. This implies that the market need not be cleared at the closing time. There are $n_{d}$ of dealers. They have transactions with their retail customers. Meanwhile dealers have wholesale transactions.

Not all of dealers are quoting their prices concurrently. They do so, if they are confident enough in their expectations. Their expectation switch between two states. Let $\{$ state 0 , state 1$\}$ be these states. Let $I_{j}$ be an index function about dealer's state of expectation. Let $I_{j}$ for $j=1,2, \ldots n_{d}$ be random variables such that

$$
I_{j}= \begin{cases}0 & \text { if in state } 0 \\ 1 & \text { if in state } 1\end{cases}
$$

If $I_{j}=0$, then the $j$ th dealer does not assume open position. If he has retail transaction, he pass it to the wholesale market. If he is in state 1 , he has reservation price and, based on it, he is ready to have open position. While in state 1 , he must be quoting buying, selling or both prices. The quoted price is equal to his reservation price. There is a unique broker in the market. Quoting price means submitting limit orders to this broker. While in state 0 , dealers do not quote prices. If they have retail transaction, they hit someone else's limit order immediately. The number of limit order prices coincides with the number of dealers who are in state 1. For a case such that one dealer quotes bid and ask, we neglect their spread. Let $N_{1}$ be the number of those in state 1 ;

$$
N_{1}=\sum_{j=1}^{n_{d}} I_{j}
$$


Random variable $I_{j}$ takes value of 1 or 0 according to two exponential distributions. The switching process of $I_{j}$ is a continuous time Markov chain consisting of two states. Sojourn time to be in state 1 follows exponential distribution with parameter $\theta_{1}$. Sojourn time in state 0 is also random variable which follows exponential distribution with parameter $\theta_{0}$. Let $P_{01}(t)$ be probability of being 1 at time $t$, starting from state 0 . This probability is obtained, for example by the method given by Ross [4] p.320.

$$
P_{01}(t)=\frac{\theta_{1}}{\theta_{0}+\theta_{1}} \exp ^{-\left(\theta_{0}+\theta_{1}\right) t}+\frac{\theta_{0}}{\theta_{0}+\theta_{1}}
$$

By equation (2),

$$
\lim _{t \rightarrow \infty} E\left[N_{1}\right]=\frac{\theta_{0} n_{d}}{\theta_{0}+\theta_{1}}
$$

\subsection{Who is quoting market bid rate ?}

When transition from state 0 to state 1 occurs, the $j$ th dealer picks his reservation price $Y_{j} . \quad Y_{j}$ 's are i.i.d.. This random variable has finite support. Rather than using $C_{j}$, we use random variable $X_{j}$ as defined below. Let $H(y)$ be (accumulative) distribution function for $Y_{j}$ :

$$
X_{j}=H\left(C_{j}\right)
$$

The random variables $X_{j}$ 's are i.i.d with uniform distribution over the unit interval.

Different values of $X_{j}$ result in long or short positions in our auction process. Transaction price coincides with the median of $X_{j}$ 's in a simple situation such that dealers trade based only on different price perspectives. Suppose that dealers arrive one by one at the market. They have reservation prices but no initial inventory. Capital gain is only motivation. Deliveries and payments are scheduled later after the auction. During the auction, long and short positions must match. Hence aggregate net position is zero. We assume that each dealer takes one transaction unit of open position. Dealers' employer exogenously impose this restriction. With such identical open positions, the number of dealers with long position matches that of short positions. In our auction process, those who have open positions submit limit orders. The prices are equal to their reservation prices. For a given time, the largest reservation price on the short side coincides with the highest buying price. Let $Z^{-}$and $Z^{+}$be the number of dealers who have short and long positions at time $t$. Net aggregate position is zero. We have following accounting relationships.

$$
\begin{aligned}
Z^{-}+Z^{+}+Z^{0} & =N_{1} \\
Z^{-}-Z^{+} & =0
\end{aligned}
$$

where

$$
Z^{0}= \begin{cases}1 & \text { if } N_{1} \text { is odd } \\ 0 & \text { if } N_{1} \text { is even }\end{cases}
$$


$Z^{0}=1$ means that one dealer has square position, which means zero inventory in FX market, although he is ready to have open position. He is quoting both buying and selling prices. This dealer's reservation price is the median of all the $X_{j}$ 's.

The highest buying price is called market bid rate. We identify the market bid as the $Z^{b}$ th smallest $X_{j}$. $Z^{b}$ coincides with $Z^{-}$if $N_{1}$ is even. Otherwise, $Z^{b}=Z^{-}+1$. Hence,

$$
Z^{b}=Z^{-}+Z^{0}
$$

Solving equation (5) and (6), $Z^{b}=\frac{1}{2}\left(N_{1}+Z^{0}\right)$. By investigating characteristics of $Z^{b}$, we can analyze volatility of the market's bid rate and hence can approximate volatility of transaction price.

Next we introduce demand and supply from the economy's fundamentals. They take a form of dealer's retail transactions. As dealers have retail transactions asynchronously, dealers have reverse transactions in the wholesale market. Let $R_{d}(t)$ and $R_{s}(t)$ be retail demand and supply from the fundamentals. They are aggregated across the dealers. They are accumulative from the morning until time $t$. Let $R(t)$ be excess demand defined as

$$
R(t)=R_{d}(t)-R_{s}(t)
$$

The dealers as a whole absorb this excess demand. At any given time, dealers' net position becomes equal to excess demand; $Z^{-}-Z^{+}=R$, where we suppressed time $t$. Accounting equations are now as follows.

$$
\begin{aligned}
Z^{-}+Z^{+}+Z^{0} & =N_{1} \\
Z^{-}-Z^{+} & =R
\end{aligned}
$$

Then, since $Z^{b}=Z^{1}+Z^{0}$, the index for the market bid is now;

$$
Z^{b}=\frac{1}{2}\left(N_{1}+R+Z^{0}\right)
$$

where

$$
Z^{0}= \begin{cases}1 & \text { if } N_{1}+R \text { is odd } \\ 0 & \text { if } N_{1}+R \text { is even }\end{cases}
$$

\subsection{Order Arrival Generations}

\subsubsection{Revisions of Expectations}

Orders consist of two types; the market orders and the limit orders. The market order hits the market bid or ask. The market bid/ask here means the maximum/minimum of the existing buying/selling limit order prices. The limit order specifies its own price. The same dealer can submit the market order while he keeps the limit order. In the following we specify stochastic mechanism to generate these two types of orders. 


\begin{tabular}{|c|c|c|c|}
\hline \multirow{2}{*}{$\begin{array}{c}\text { action upon } \\
\text { entry into state 1 }\end{array}$} & $X_{j}<$ bid & bid $<X_{j}<$ ask & ask $<X_{j}$ \\
\hline $\begin{array}{c}\text { consequent } \\
\text { position }\end{array}$ & quoting & bid and ask & hitting ask \\
\hline $\begin{array}{c}\text { accompanying } \\
\text { limit order price }\end{array}$ & buying price & square & long \\
\hline
\end{tabular}

\section{Table 1: Picked Reservation Price and Accompanying Actions}

The sequence of orders are generated by two sources. The first source is dealers' revisions of expectations. The second source is retail transactions. The process of revision of expectations consists of two parts; a process of switching between two states and a process of choosing reservation price. The $j$ th dealer has a random variable $I_{j}(t)$ of equation (1). It start with $I_{j}(0)=0$ for all of dealers. The sojourn time $s$ in state $i$ has an exponential distribution. Its parameter is $\theta_{i}$. When $I_{j}$ changes value from 0 to 1 , i.e. entering state 1 , he picks up his reservation price, $X_{j}$. This $X_{j}$ is a random variable. It is uniformly distributed over unit interval. When he leaves state 1 , i.e. when $I_{j}$ switching to 0 , he abandons $X_{j}$.

Table (1) summarizes actions upon entry into state 1 . When a dealer picks $X_{j}$, he compares it with market bid and ask. If $X_{j}>a s k$, then he hits ask. He buys spot FX at ask. He comes to have long position. Then he submits his reservation price as his selling price. He expects transaction price will reach his selling price. He waits with this limit order. If $X_{j}<$ bid when entering state 1, then he hits bid. He has short position and waits with his limit order. His buying price is equal to his reservation price. If bid $<X_{j}<a s k$, then he wouldn't hit either bid or ask. Instead he quotes his reservation price as both of bid and ask. When he leaves state 1 , he abandons his $X_{j}$ and corresponding position. If he has long or short position then, he hits market bid or ask to close position. Thus switching between states give rise to the generation of arrivals of orders. The switching process of $I_{j}$ generates volume and choosing process of $X_{j}$ sorts it out between buying and selling. We assume that $\mathrm{bid} /$ ask spread is negligible, if quoted by the same dealer.

\subsubsection{Retail Transactions}

Retail demand and supply $R_{d}(t)$ and $R_{s}(t)$ are accumulative quantities which retail customers bought from and sold to dealers until time $t$. They are sums over all the dealers. The retail transactions appear as order flows in the wholesale market. $R_{d}(t)$ and $R_{s}(t)$ have Poisson distributions with parameter $\lambda_{d}$ and $\lambda_{s}$.

$$
R(t)=R(0)+\left(\lambda_{d}-\lambda_{s}\right) t
$$

Constructions of $R_{d}(t)$ and $R_{s}(t)$ are as follows. 
Dealer's objective is daily profit maximization. He has retail customers. No marketing effort is made. He trades with them anytime they want during business hours. His profit is constant per transaction. Their arrivals constitute two Poisson processes; one for customer's buying and the other selling. Quantity of a retail arrivals is one transaction unit. These retail arrivals immediately change into Poisson arrivals of order flows in the wholesale market. Such conversion is due to the following assumption (p1) to (p3).

(p1) Dealer is risk neutral.

(p2) There is a restriction on the maximum size of position; one transaction unit.

(p3) Exceeding the restriction on the position due to retail transaction is allowed but only for a moment.

If he is in state 1 , he must have constructed open position. Because of (p1), the open position must be at its maximum. So (p2) must have been binding. Meanwhile anytime he may have a retail transaction. Had this occurred, he counterbalances the retail transaction by hitting the market bid or ask. This is by (p3). The retail arrivals become Poisson arrivals in the wholesale market by the immediate counterbalancing. If dealer is in state 0 , he does not want to assume open position. By this reason, he counterbalances retail transaction. Poisson arrivals pass through to the market. Sum of Poisson's is also Poisson. Having aggregated across dealers, $R_{d}$ and $R_{d}$ are also Poisson variables.

Excess demand $R$ is defined as equation(9). Dealers as a whole absorb this excess demand. We introduce an assumption on the value of $R$. Namely, $R$ cannot be bigger than dealers' aggregate open position; for a given $N_{1}$,

$$
-N_{1} \leq R \leq N_{1}
$$

If $R=N_{1}$, then it means that all the dealers in state 1 have short positions.

\subsection{Markov Transitions of Market Inventory States}

\subsubsection{Transition Intensities}

A pair of $N_{1}$ and $R$ determine aggregated dealers' position. Minus valued inventory equals to the excess demand $R$. The combinations of $N_{1}$ and $R$ constitute a set of such net inventories. We call this set of net inventory and denote by $\Omega$. We call its element an inventory state Let $\omega(n, r)$ be an inventory state such that $N_{1}=n$ and $R=r$.

$$
\Omega=\{\omega(n, r)\}
$$

For each value of $N_{1}, R$ takes integer values such that $-N_{1} \leq R \leq N_{1}$. For a given $N_{1}$, there are $2 N_{1}+1$ of $R$ 's values. And $N_{1}=0,1, \ldots, n_{d}$. Hence, $\Omega$ contains

$$
\Sigma_{N_{1}}^{n_{d}}=\left(n_{d}+1\right)^{2}
$$




\begin{tabular}{|c|c|c|c|c|c|c|c|}
\hline $\begin{array}{c}\text { inventory } \\
\text { state }\end{array}$ & $\omega(n-1,0)$ & $\ldots$ & $\omega(n,-1)$ & $\omega(n, 0)$ & $\omega(n, 1)$ & $\ldots$ & $\omega(n+1,0)$ \\
\hline $\begin{array}{c}\text { column } \\
\text { number }\end{array}$ & $\xi_{n-1}$ & $\ldots$ & $\xi_{n}-1$ & $\xi_{n}$ & $\xi_{n}+1$ & $\ldots$ & $\xi_{n+1}$ \\
\hline intensity & $n \theta_{1}$ & 0 & $\lambda_{s}$ & $*$ & $\lambda_{d}$ & 0 & $\left(n_{d}-n\right) \theta_{0}$ \\
\hline
\end{tabular}

Table 2: Intensities to Exit from $\omega(n, 0)$ and to Enter Other States When the market exits from $\omega(n, 0)$, it enters into either $\omega(n-1,0), \omega(n,-1), \omega(n, 1)$ or $\omega(n+1,1)$. Elements left of the $\xi_{n-1}$ th are 0 . Also they are 0 right of the $\xi_{n+1}$ th column, where $\xi_{j}=(j-1)^{2}+(j+1)$ for $j=n-1, n, n+1$. The exit has negative intensity; $*=-\left\{\left(n_{d}-n\right) \theta_{0}+n \theta_{1}+\lambda_{d}+\lambda_{s}\right\}$. The sum of the $\xi_{n}$ th row is zero.

of $\omega$ 's. Processes for $N_{1}$ and $R$ determine transition intensities between $\omega$ 's. Let $Q$ be infinitesimal operator defined on $\Omega$. By equation (16), it is a $\left(n_{d}+1\right)^{2} \times\left(n_{d}+1\right)^{2}$ matrix.

We construct $Q$ matrix as follows. Element $Q(i, j)$ shows transition intensity from the $i$ th into $j$ th state. We arrange $\omega$ 's from those with smaller $N_{1}$ and, for each $N_{1}$, we put them from $R=-N_{1}$ to $R=N_{1}$. Table (8) in Appendix shows the correspondences between $\omega$ 's and locations in $Q$ matrix.

The $i$ th row of $Q$ lists the intensities of exiting $i$ th state and entering others. We consider transitions from $\omega(n, 0)$ to other states as an example. They are in the $n^{2}+n+1$ th row. Table (8) in Appendix shows correspondences between inventory states and their locations in $Q$ matrix. Let $\xi_{n}=n^{2}+n+1$ to simplify notation. Table (2) lists transition intensities from $\omega(n, 0)$. Their derivations are given in the following. The example is for the cases such that $N_{1}<n_{d}$ and $R \neq \pm N_{1}$. Other cases are in Appendix.

Element $Q\left(\xi_{n}, \xi_{n}\right)$ shows intensity to depart from $\omega(n, 0)$. Exit from $\omega(n, 0)$ occurs when $N_{1}$ or $R$ changes value. The market moves into one of the following states; $\omega(n-1,0), \omega(n,-1), \omega(n, 1)$, and $\omega(n+1,0)$. If $N_{1}=n$, the one of the $N_{1}$ dealers in state 1 may switch to state 0 . Sojourn time in state 1 has an exponential distribution with parameter $\theta_{1}$. There are $n$ of i.i.d. sojourn times. So the intensity is $n \theta_{1}$. With this the market moves to $\omega(n-1,0)$. As for $\omega(n, 1), \Delta R=+1$ is Poisson arrival. Its intensity is $\lambda_{d}$. With this intensity, the market moves to $\omega(n, 1)$. And similar arguments hold for $\omega(n+1,0)$ and $\omega(n,-1)$.

\subsubsection{Transition Probabilities}

For a given infinitesimal operator $Q$, we can obtain transition probability matrix $P(t)$ by solving Kolmogrov's backward equation:

$$
P^{\prime}(t)=Q P(t)
$$


The solution is given by

$$
P(t)=e^{Q t}
$$

Exponential notation of matrix implies that $e^{Q t}=I+Q t+\frac{1}{2} Q^{2} t^{2}+\frac{1}{3 !} Q^{3} t^{3}+\ldots$ The elements on the $(i, j)$ th row of $P(t)$ tells the probability to be the $j$ th state after time $t$ elapsed, starting with $i$ th state. Equation (18) converges to stationary probabilities as $t \rightarrow \infty$.

\subsection{Distributions of Market Bid Rates}

\subsubsection{Distribution of Bid Rate on $\mathrm{P}$ interval}

We have three finite intervals to define reservation prices. They are $P, S$ and $C$ intervals. $P$ interval is unit interval $[0,1]$. Reservation price $X_{j}$ is uniformly distributed on $P$. Random variable $X_{j}$ is mapped on another unit interval $S$ by function $J(x)$. Then it is mapped on finite $C$ interval; $\left[\gamma_{0}, \gamma_{1}\right]$ where $\gamma_{0}, \gamma_{1}>0$. From $X_{j}$ we construct $Y_{j}$ and $C_{j}$ as follows.

$$
Y_{j}=J\left(X_{j}\right)
$$

where $J(x)$ is piecewise continuous, $J^{\prime}(x)>0$ where is differentiable. And that $J(0)=0$, and $J(1)=1$.

$$
C_{j}=\gamma_{0}+\gamma_{1} Y_{j} \quad \text { where } \gamma_{0}, \gamma_{1}>0
$$

We call the $j$ th smallest reservation prices $C_{j}$ where $j=1, \ldots, N_{1}$. $C_{j}$ 's are i.i.d. They have denomination of the currency. The support for $C_{j}$ is positive finite interval $\left[\gamma_{0}, \gamma_{1}\right]$. Random variable $Y_{j}$ is the standardized value of $C_{j} . Y_{j}$ has a distribution function $H(y) ; H(y)=\operatorname{Pr}\left(Y_{j} \leq y\right)$. Random variable $X_{j}$ is constructed from $Y_{j}$, using $H(y)$;

$$
X_{j}=H\left(Y_{j}\right) .
$$

where $X_{j}$ is uniformly distributed on $[0,1]$. Function $J(x)$ in equation (19) is an inverse function of $H(y)$.

We denote bid rates on $P, S$ and $C$ intervals by $X, Y$ and $C$, dropping subscript. Notations are summarized in table 2.5.1. We investigate volatility of $C$ on $S$ interval. The change in volatility is attributable to that of $Y$ as well as coefficient $\gamma_{1}$ of equation (20).

The infinitesimal operator $Q$ determines transition probabilities between the inventory states, for a given time interval. For a given the market inventory state $\omega$, the bid rate $X$ is the $Z^{b}$ th reservation price from the smallest by equation(12). If $N_{1}+R=2 \kappa-1$, for positive integer $\kappa$, then $Z^{b}=\kappa$ by eq (12). The bid rate $X$ has Beta distribution. For $N_{1}=n$, its density is given by

$$
f(x)=\frac{\Gamma(n+1)}{\Gamma(\kappa) \Gamma(n-\kappa+1)} x^{\kappa-1}(1-x)^{(n-\kappa+1)-1}
$$




\begin{tabular}{|c|c|c|}
\hline $\begin{array}{c}\text { reservation } \\
\text { prices }\end{array}$ & $\begin{array}{c}\text { bid } \\
\text { rate }\end{array}$ & support \\
\hline$X_{j}$ & $X$ & $P$ interval; $[0,1]$ \\
\hline$Y_{j}$ & $Y$ & $S$ interval; $[0,1]$ \\
\hline$C_{j}$ & $C$ & $\begin{array}{r}\text { positive finite } C \text { interval; } \\
{\left[\gamma_{0}, \gamma_{0}+\gamma_{1}\right]}\end{array}$ \\
\hline
\end{tabular}

Table 3: Bid Rates on Three Intervals where $j=1, \ldots N_{1}$ and $0<\gamma_{0}, \gamma_{1}$ There are $N_{1}$ of reservation prices. They are renumbered from the smallest.

This is $\operatorname{Beta} \operatorname{density}(\kappa, n-\kappa+1)$, where $N_{1}=n$. If $N_{1}+R=2 \kappa$, then the density of bid rate is also given by equation (22).

Next, we calculate moments of $Y$ on $S$ interval. Since $Y=J(X)$, we can obtain them as $E[J(X)]$ for $k=1,2$ :

\subsection{Dealer's Expectation Formation}

In this subsection, we show how representative dealer can form expectation using our model. He forms expectation, knowing that dealers' expectations differ each other. Dealers may agree on the price determination mechanism but still they end up with having different price perspectives.

He observes bid rate samples on $\mathrm{C}$ interval; $C(t)$. The distribution of reservation prices $C_{j}$ is not observable. Nor are parameters of two order generation Processes. He can observe only his own retail transactions for sure. His present action depends on FLE, the first local extremum, of $C(t+s)$ for $s>0$. Forming expectation on $C(t+s)$ requires estimates on $x(t), y(t)$, and parameters of equation (19) and (20). Let stared variables and a funcion be his estimates of the original ones; for example, $J^{*}(x)$ for $(19)$ and $\gamma_{0}^{*}$ and $\gamma_{1}^{*}$ for $(20)$

a. Now we are time $t$. He wants to estimate $Z^{b}$ of (12). He fix $E\left[N_{1}(t+s)\right]=n^{*}$ for $s>0$ and $Z^{0}=0$.

b. He wants to estimate $R(t+s)$ for $s>0$. Its expected value is given by equation (14). $\lambda_{d}$ and $\lambda_{s}$ may not be stationary. This gives rise to FLE. He thinks unbalanced arrivals would last intra-day, for the duration of $\tau$ from now on. He substitutes $R^{*}(t)$ and $\left(\lambda_{d}^{*}-\lambda_{s}^{*}\right) \tau$ into equation (14). After time $\tau$, he thinks that arrivals may be either balanced or reversed. He calculates $R^{*}(t+\tau)$; FLE of $R(t+s)$ for $s>0$. Let $\Delta R^{*}$ be $\Delta R^{*}=\left(\lambda_{d}^{*}-\lambda_{s}^{*}\right) \tau$ Let the double stared be FLE value; $R^{* *}=R^{*}(t+\tau)$. Using simplifying notations,

$$
R^{* *}=R^{*}(t)+\Delta R^{*}
$$


c. He substitutes $R^{* *}$ and $n^{*}$ into (12). He obtains $Z^{b * *}$.

$$
Z^{b * *}=Z^{b *}+\frac{\Delta R^{*}}{2}
$$

d. For a given pair of $Z^{b}$ and $N_{1}$, the density of bid rate $X$ on $P$ interval is given by

$$
\frac{\Gamma\left(N_{1}+1\right)}{\Gamma\left(Z^{b}\right) \Gamma\left(N_{1}-Z^{b}\right)} x^{Z^{b}-1}(1-x)^{\left(N_{1}-Z^{b}+1\right)-1}
$$

Hence, the expected value of this Beta distribution is given by

$$
E[X]=\frac{Z^{b}}{N_{1}} .
$$

He substitutes $Z^{b * *}$ and $N_{1}=n^{*}$ into equation (26). Then he obtains his FLE on $P$ interval.

$$
x^{* *}=\frac{1}{n^{*}}\left(z^{b *}(t)+\frac{\Delta R^{*}}{2}\right)
$$

e. He substitutes $x^{* *}$ into equations (19) and (20).

$$
c^{* *}=\gamma_{0}^{*}+\gamma_{1}^{*} J^{*}\left(x^{* *}\right)
$$

If $J^{*}(x)$ is linear in the relevant range, then

$$
c^{* *}=C(t)+\gamma_{1}^{*} J^{*}\left(\frac{\Delta R^{*}}{2 n^{*}}\right)
$$

This is his FLE on $C$ interval.

Only observable variable is bid rate on $C$ interval. Therefore dealers may agree on the price determination mechanism. They are likely to have different estimates on the parameters. The heterogeneity of expectations persists.

\section{Variability of Volume and Volatility}

\subsection{Approximating Volatility}

Our volatility is the variance of equally spaced samples of bid rate. We approximate changes in the transaction price volatility by the bid rate. There are always bid and ask. One of them randomly becomes transaction price. Therefore, transaction price volatility consists of two parts; the first due to bid/ask spread and the second due to movements of their levels. Bid and ask, though not simultaneously, tend to move together. We assume the first part of the volatility is constant for a given day. We focus on the second part. We approximate its change by change in the bid rate volatility. 


\begin{tabular}{|c|c|}
\hline \multicolumn{2}{|c|}{$\lambda_{d}$ and $\lambda_{s} \uparrow$} \\
\hline volume & volatility \\
\hline+ & + \\
& volume effect \\
\hline
\end{tabular}

Table 4: Larger Retail Transactions: $\operatorname{Var}(R)=\lambda_{d}+\lambda_{s}$ where $\lambda_{j}=\operatorname{Var}\left(R_{j}\right)$ for $j=d, s$.

\begin{tabular}{|c|c|}
\hline \multicolumn{2}{|c|}{$\theta_{0}$ and $\theta_{1} \uparrow$} \\
\hline volume & volatility \\
\hline+ & + \\
cycle effect & convergence effect \\
\hline
\end{tabular}

Table 5: Shorter Expectation Revision Cycle: The expected cycle length to go through two expectation states is given by $\frac{1}{\theta_{0}}+\frac{1}{\theta_{1}}$

\begin{tabular}{|c|c|}
\hline \multicolumn{2}{|c|}{$\frac{\theta_{0}}{\theta_{0}+\theta_{1}} \downarrow$} \\
\hline volume & volatility \\
\hline+ & + \\
if $\frac{1}{\theta_{0}}+\frac{1}{\theta_{1}} \uparrow$ & thin market effect \\
\hline
\end{tabular}

Table 6: Smaller $E\left[N_{1}\right]$,i.e., Thin Market : $E\left[N_{1}\right]=\frac{\theta_{0} n_{d}}{\theta_{0}+\theta_{1}}$

\begin{tabular}{|c|c|}
\hline \multicolumn{2}{|c|}{$\alpha \uparrow$ and $/$ or $\gamma_{1} \uparrow$} \\
\hline volume & volatility \\
\hline no change & + \\
& heterogeneity effect \\
\hline
\end{tabular}

Table 7: More Heterogeneous Expectations: $\alpha$ increases volatility of bid rate on $S$ interval and, for a given $\alpha, \gamma_{1}$ increases that on $C$ interval. 


\subsection{Causes and Effects}

Parameters of two order generating sources influence volatility as well as trading volume. Dispersion of reservation prices also influences volatility. Causes and effects are summarized in Tables (4) to (7). Prices change enough to absorb unbalanced arrivals of retail transactions. Dealers as a whole absorb them with aggregate open position. As $R$ fluctuates more, volatility increases. Also as the reservation prices are dispersed thinly and/or on a wider range, then volatility increases.

Volume Effect: The first source of the order flows is retail transactions. As the retail transactions increase, volatility increases. We call such consequence "volume effect". This is consequence of stochastic property of $R$. Random variable $R(t)$, as defined in equation (9), is difference of two Poisson variables. Its expected value is $\left(\lambda_{d}-\lambda_{s}\right) t$ and its variance is $\left(\lambda_{d}+\lambda_{s}\right) t$. Let $N$ denote random variable with standard normal distribution. Then for $R(0)=0$

$$
\frac{R(t)-\left(\lambda_{d}-\lambda_{s}\right) t}{\sqrt{\left(\lambda_{d}+\lambda_{s}\right) t}} \Rightarrow N
$$

By the central limit theorem, the difference of two Poisson variables converges to a normal distribution and the approach to normalty is rapid. ( Johnson,Kotz \& Kemp [5] p.191). Our $R$ has both tails truncated; condition of equation (15); $-N_{1} \leq R \leq$ $N_{1}$. As $N_{1}$ increases, we have better approximation. As $\lambda_{d}$ and $\lambda_{s}$ increase, trading volume increases. At the same time, $R$ come to have larger variance. For a given $N_{1}, Z^{b}$ changes more. And hence, $\operatorname{Var}[Y]$ and $\operatorname{Var}[C]$ increase. We have volume effect.

Convergence Effect: The second source of order arrivals is the expectation revisions. This process has two parameters ; $\theta_{0}$ and $\theta_{1}$. The dealer's sojourn times in state 0 and state 1 are exponentially distributed. The expected sojourn times are given by $\frac{1}{\theta_{0}}$ and $\frac{1}{\theta_{1}}$. As $\theta_{0}$ and $\theta_{1}$ become smaller, frequent revisions increases trading volume. We call this consequence "cycle effect" on trading volume. The frequent revisions speed up transitions. Transition probability $\operatorname{Pr}(\omega(m, l)$ at $t+$ $s \mid \omega(n, r)$ at t) converges faster to the stationary probability $\pi_{s}(m, l)$. For a given finite time interval and $N_{1}=n$, the probabilities to reach far away values of $N_{1}$ increase. Volatility increases. The volatility in this case is the one calculated for short interval of time. We call such "convergence effect". As the time interval to take equally spaced samples becomes longer, this effect disappears.

Thin Market Effect: As shown in equation (3), relative size of $\theta_{0}$ to $\theta_{1}$ changes the expected number of dealers in state 1 . This ratio is the thickness of the market. As $\theta_{0}$ becomes relatively smaller, the market becomes thin. Volatility increases. We call this "thin market effect" on volatility.

Heterogeneity Effect: As the reservation prices spread out wider, the required price change to absorb a given amount of unbalanced retail transactions becomes 
larger. We call such causality "heterogeneity effect". Let $\tau$ be unit time interval. We like to show this effect as an increase in the following value.

$$
\operatorname{Var}[J(X(\tau))-J(X(0))] .
$$

We need to specify the density of the reservation prices. It will be $h(y)$ of equation(32) - Tractability of its (accumulative) distribution function $H(y)$ and the latter's inverse function depend on it. We choose a linear combination of two uniform distributions for $Y_{j}$ 's density. This functional form makes $H(y)$ peace-wise continuous. With this linear function, we can still approximate various patterns of dispersion. Meanwhile, $J(X)$ is tractable. We introduce an example of $Y_{j}$ 's density in the next subsection. With these, we show by approximation that, as the reservation prices become more heterogeneous, the value of equation(31) increases and hence the variance of bid rate increases.

\subsection{Volatility on $S$ Interval}

\subsubsection{Distribution of Reservation Prices on $S$ Interval}

We express the density for reservation price, $Y_{j}$, as a weighted sum of two uniform distributions. We use uniform distribution for the sake of tractability of $J(x)$

$$
h(y)=\alpha h_{1}(y)+(1-\alpha) h_{2}(y)
$$

where $\alpha>0$. The terms on RHS are given by

$$
\begin{array}{lr}
h_{1}(y)=1 & \text { for } y \in[0,1] \\
h_{2}(y)=\frac{1}{\beta_{2}-\beta_{1}} & \text { for } y \in\left[\beta_{1}, \beta_{2}\right]
\end{array}
$$

where $1>\beta_{2}>\frac{1}{2}>\beta_{1}>0$. Since equation(32) consists of The uniform distributions, $H(y)$ and $J(x)$ become piecewise linear. Function $J(x)$ of eq $(19)$ is the inverse function of (accumulative) distribution function of $Y_{j}$. By changing values of $\alpha, \beta_{1}$ and $\beta_{2}$, the density $h(y)$ takes a form from a spike-like to $\mathrm{U}$ shape. For $\alpha>1$, it looks $\mathrm{U}$ shape. To keep equation (32) from becoming negative, these parameters must satisfy

$$
0<\alpha<\frac{1}{1-\left(\beta_{2}-\beta_{1}\right)} .
$$

Then we obtain the distribution function for $Y_{j} ; H(y)$.

$$
\mathrm{H}(y)= \begin{cases}\alpha y & \text { for } y \in\left[0, \beta_{1}\right] \\ \alpha y+(1-\alpha) \frac{y-\beta_{1}}{\beta_{2}-\beta_{1}} & \text { for } y \in\left[\beta_{1}, \beta_{2}\right] \\ \alpha y+1-\alpha & \text { for } y \in\left[\beta_{2}, 1\right]\end{cases}
$$

Let $J(x)$ be an inverse of $\mathrm{H}(y)$ such that, for $0 \leq x \leq 1$,

$$
J(x)=J_{1}(x)+J_{2}(x),
$$


where terms on RHS are given by and

$$
\begin{gathered}
J_{1}(x)=\frac{1}{\alpha} x \quad \text { for } x \in[0,1] \\
J_{2}(x)= \begin{cases}0 & \text { for } x \in A_{1} \\
\frac{1-\alpha}{\left(\beta_{2}-\beta_{1}\right) \alpha+1-\alpha}\left(-\frac{x}{\alpha}+\beta_{1}\right) & \text { for } x \in A_{2} \\
J_{2}\left(\alpha \beta_{2}+1-\alpha\right) & \text { for } x \in A_{3}\end{cases}
\end{gathered}
$$

where $A_{1}=\left[0, \alpha \beta_{1}\right], A_{2}=\left[\alpha \beta_{1}, \alpha \beta_{2}+1-\alpha\right], A_{3}=\left[\alpha \beta_{2}+1-\alpha, 1\right]$.

Then we can calculate $E[Y]$ as $E[J(X)]=E\left[J_{1}(x)\right]+E\left[J_{2}(x)\right]$. Since $X$ has Beta distribution $(22), E\left[J_{1}(X)\right]=\frac{\kappa}{\alpha(n+1)}$. And $E\left[J_{2}(X)\right]$ is obtained as a sum of incomplete beta integrals; one for $A_{2}$ and the other for $A_{3}$. In later subsection, by changing value of $\alpha$, we describe the effect of heterogeneity of reservation prices.

Equation (38) and (39) have liner forms. Yet equation (31)is not yet tractable. We approximate eqn(31) as in the following.

\subsection{Movements between On-Average Locations}

We use representative value of $Y$ for each inventory state. For a given inventory state $\omega(n, r)$, let $\mu(n, r)$ be an expected value of $J(X)$;

$$
\mu(n, r) \equiv E[J(X) \mid \omega(n, r)]
$$

where $n=0,1, \ldots, n_{d}$ and $r=-n+1, \ldots, 0,1, \ldots, n$. We do not have $r=-n$ in the above. The inventory state $\omega(n,-n)$ does not have bid rate. If $R=-N_{1}$, everybody is quoting selling price. Starting with the initial state of $\omega(n, r)$, after $\tau$ elapse, the market inventory state moves to $\omega(m, l)$. We compare the on-average locations of $Y ; \mu(m, l)-\mu(n, r)$.

Let $q_{t}(m, l \mid n, r)$ be transition probability from $\omega(n, r)$ to $\omega(m, l)$ during time interval $[0, \tau]$. For a given time, the market is in the inventory state $\omega(n, r)$ with stationary probability $\pi_{s}(n, r)$. Whichever state you may start with, $\pi_{s}(n, r)$ is probability to be in $\omega(n, r)$ on average as $t \rightarrow \infty$. It is the limit of equation (18). For a given $\omega(n, r)$ where $r \neq-n$, we define the following random variable. For $m=1,2, \ldots, n_{d}$ and $l=-m+1, \ldots, m$

$$
\begin{array}{rlr}
W_{n, r} & =\mu(m, l)-\mu(n, r) & \text { with } q(m, l \mid n, r) \\
W & =W_{n, r} & \text { with } \pi(n, r)
\end{array}
$$

where, for $r>-n$,

$$
\begin{aligned}
q(m, l \mid n, r) & =\frac{q_{t}(m, l \mid n, r)}{\sum_{k>-j} q_{t}(j, k \mid n, r)} \\
\pi(n, r) & =\frac{\pi_{s}(n, r)}{\sum_{l>-m} \pi_{s}(m, l)}
\end{aligned}
$$


In the above, we adjusted probabilities after excluding the case of $\omega\left(N_{1},-R\right)$. We approximate equation (31) by $\operatorname{Var}[W]$.

$$
\operatorname{Var}[W]=\sum_{n=1}^{n=n d} \sum_{r=-r+1}^{r=n} E\left[W_{n, r}^{2}\right] \pi(n, r)-\left\{\sum_{n=1}^{n=n d} \sum_{r=-n+1}^{r=n} E\left[W_{n, r}\right] \pi(n, r)\right\}^{2}
$$

In the next subsection we use the equation (45) to show effects of the relevant factors on volatility.

\subsection{Example of Heterogeneity Effect}

We like to show the value of equation (45) increases as the reservation prices become more heterogeneous. Deriving this result only through qualitative argument is, however, difficult. We used a numerical example. By approximation, we obtained the heterogeneity effect. For the sake of tractability, we assume three simplifying conditions as shown below. And our numerical examples are $n_{d}=10$ and $\lambda_{d}=\lambda_{s}=$ 2.5 .

(a1) $\lambda_{d}=\lambda_{s}$

(a2) $\beta_{2}=1-\beta 1$ where $0<\beta_{1}<0.5$

(a3) $\alpha \beta_{1}=\epsilon$, where $0<\epsilon<0.5$

The implications are as follows.

(a1): Random variable $R$ is difference of two Poisson variables as defined by equation (9). By (a1), $E[R]=0$. Assumption (a1) makes it possible for each inventory state to have positive stationary probability. In addition, together with central limit theorem of (30), for given $n$ and $r$, we have

$$
\pi(n, r)=\pi(n,-r)
$$

$R$ changes on its own except when $R= \pm N_{1}$. So we use the following approximation.

$$
q(m,-l \mid n,-r) \approx \operatorname{Pr}\left(N_{1}(t)=m \mid N_{1}(0)=n\right) \operatorname{Pr}(R(t)=l \mid R(0)=r)
$$

We apply normal distribution to evaluate $\operatorname{Pr}(R(t)=l \mid R(0)=r)$ as $\operatorname{Pr}(\Delta R(t)=$ $l-r)$. By $($ a1 $)$, for a given $r, \operatorname{Pr}(\Delta R(t)=r)=\operatorname{Pr}(\Delta R(t)=-r)$. This symmetry is used to evaluate the second term of equation (45).

(a2): The density $h(y)$ of equation (32) has line symmetry. The line of symmetry goes through $y=0.5$. Meanwhile, each market inventory state has the density for bid rate on $P$ interval, $X$. Among these densities, there are pairs with line symmetry 
each other. Table (9) in the appendix shows list of such pairs. Expected values of $Y$ of these pairs sum up to 1 . This symmetry remains when we change degree of reservation price heterogeneity. They simplify evaluation of the second term of equation (45).

(a3): As we change value of $\alpha$ while keeping (a3), reservation price density of $h(Y)$ changes from a spike in the middle to two spikes on both ends. Since $\beta_{1}<0.5$, for a given $\epsilon<0.5$, it must be that $\alpha>\frac{\epsilon}{\beta_{1}}$. When $\alpha \approx \frac{\epsilon}{\beta_{1}}, h(y)$ looks like a spike at $y=0.5$. When $\alpha=1$, then $h(y)$ is horizontal line. When $\alpha>1, h(y)$ has a dent in the middle. The tails with squire shape on both ends have the same probability. Together with (a2), substituting (a3) into equation(39), $J(x)$ has kinks at $x=\epsilon$ and $1-\epsilon$ as is shown in Figure(1). Parameter $\alpha$ signifies degree of dispersion of reservation prices in equation (32). And $\alpha$ becomes only variable when we calculate expected value of equation (39). If value of equation (45) increases as $\alpha$ increases, then we have heterogeneity effect.

We used numerical examples of $n_{d}=10$ and $\lambda_{d}=\lambda_{s}=2.5$ and approximated equation (45). The result confirmed heterogeneity effect. The details of approximation are given in Appendix.

The value of $\alpha$ is not the only parameter for the heterogeneity. While $\alpha$ sets degree of heterogeneity on $S$ interval, $\gamma_{1}$ in equation (20) sets degree of the heterogeneity on $C$ interval. For a given value of $\operatorname{Var}[W]$, as $\gamma_{1}$ increases, $\operatorname{Var}[C]$ increases. The parameter $\gamma_{1}$ also causes heterogeneity effect. @

\section{Conclusion}

We describe brokered foreign exchange auction as a mechanism which consists of continuous time stochastic processes. Our approach makes volume and volatility endogenous. Heterogeneity of expectations, order arrivals from the retail customers interact. These two key elements and the dealers' revising of expectations determine trading volume and volatility. We show causes of variability of volume and volatility. However, it is difficult to show the effect of heterogeneous expectations completely in an analytical way. We used numerical example to confirm it.

Our model provides what has been lacked in the existing microstructure literature. First, our use of arrival intensities makes it possible to analyze continuous auction. It is an awkward problem to defining equilibrium for continuous auction. Our approach distinguishes expected value and realized value. Constant expected value and fluctuated observed value are not contradictory. Second our use of FLE, the first local extremum avoids the problem how to define junk value when we do not have a model to set equilibrium. Third our model is about the processes which generate order arrivals. These processes fill what is missing in the order flow analysis of Lyon[1]. 
Summary of price determination mechanism are as follows. Two sources generate order arrivals; processes of the expectation revisions and of the retail transactions. The arrivals are asynchronous. The retail transactions are not balanced concurrently. Dealers have heterogeneous expectations. So are their reservation prices. Because of this heterogeneity, dealers as a whole absorb the unbalanced arrivals. For a given amount of the unbalanced, how much price has to change determines volatility. As the reservation prices are more dispersed, the required change becomes larger. Volatility increases.

We identify causes of volatility change as follows. Volatility of transaction price consists of two parts; the first one due to bid/ask spread and the second due to movements of bid/ask levels. We approximate the change in transaction price volatility by that of the bid rate. We derive bid rate's stochastic properties. As the expectations become more heterogeneous, the volatility increases. The retail transaction's volume also influences volatility. The expectation revisions also influence volume and volatility. Thus volume and volatility are endogenous. Correlation between volume and volatility is variable. This variability is attributable to the effects of the relevant factors as shown in Table (4) to (7). As for empirical applicability, our model makes volume and volatility endogenous so that it is possible to reconcile changing correlation between volume and volatility.

Features of our model are as follows: (i) We use stochastic processes of arrivals of buyers and sellers to handle asynchronous order arrivals. (ii) We distinguish two sources of order generations. (iii)We describe interactions between order arrivals as the continuous time Markov transitions between the states of the market. We derive an infinitesimal operator which governs Markov transitions so that it becomes possible to find transition probabilities. (iv) The benchmark value which, for the sake of dealer's decision making, substitutes an expected equilibrium. (v) Our model itself can be a mechanism for a dealer to form his own price expectation while taking into account of distribution of others.'

\section{REFERENCES}

[1] R.K. Lyons, The Microstructure approach to exchange rates (Cambridge, MA: The MIT Press, 2001)

[2] M.B. Garman, Market Microstructure, Journal of Financial Economics, 3, 1976, 257-275

[3] Y. Amihud \& H. Mendelson, Dealership Market, Market-making with

Inventory, Journal of Financial Economics, 8, 1980, 31-53

[4] S. M. Ross, Introduction to probability models, sixth edition ( San Diego, CA: Academic Press, 1997)

[5] N.L. Johnson, S. Kotz \& A. W. Kemp, Univariate discrete distributions, 2nd ed. (New York, John Wiley \& Sons, 1993) 190-192 


\begin{tabular}{|c|c|c|}
\hline$N_{1}$ & $\begin{array}{c}\text { inventory } \\
\text { states }\end{array}$ & $\begin{array}{c}\text { column and row } \\
\text { numbers }\end{array}$ \\
\hline \multirow{3}{*}{$n-1$} & $\omega(n-1,-n+1)$ & $(n-1)^{2}+1$ \\
& $\ldots$ & $\ldots$ \\
& $\omega(n-1, n-1)$ & $n^{2}$ \\
\hline \multirow{4}{*}{$n$} & $\omega(n,-n)$ & $n^{2}+1$ \\
& $\omega(n, 0)$ & $n^{2}+n+1$ \\
& $\ldots$ & $\cdots$ \\
& $\omega(n, n)$ & $(n+1)^{2}$ \\
\hline
\end{tabular}

Table 8: Market Inventory States and Their Locations in $Q$ matrix For a given $N_{1}=n$, there exist $2 n+1$ of inventory states. $\omega(n, 0)$ is located $(2 n+1)^{2}-n$ th row and column.

\section{A Construction of $Q$ Matrix}

\section{A.1 Locations of States in $Q$ Matrix}

For a given $N_{1}=n$, there are $2 n+1$ states; $R=-n \ldots 0 \ldots n$. A set of inventory states with $N_{1}=n$ ends at $(n+1)^{2}$ textitthrow and column in $Q$ matrix;and

$$
\sum_{j=0}^{n}(2 j+1)=(n+1)^{2}
$$

Their corresponding row numbers are as in table (8). Since $N_{1}=0, \ldots, n_{d}$ and equation(48), the size of $Q$ matrix is given by $\left(n_{d}+1\right)^{2}$.

\section{A.2 Values of Element of $Q$ Matrix}

Random variable $R$ takes value between $-N_{1}$ and $N_{1}$. When the market is in $\omega(0,0)$, the only possible change is $\Delta N_{1}=+1$; moving to $\omega(1,0)$. Sojourn time in state 0 has an exponential distribution with parameter $\theta_{0}$. since all of dealers are state 0 , there are $n_{d}$ of i.i.d. random variables. The intensity to exit is given by $n_{d} \theta_{0}$. Hence, $Q(1,1)=-n_{d} \theta_{0}$ and $Q(1,3)=n_{d} \theta_{0}$. All other elements on the first row are zero.

Similarly the last row of $Q$ have only two non-zero entry. The $\left(n_{d}+1\right)^{2}$ th row corresponds to $\omega\left(n_{d}, n_{d}\right)$. Only possible change is $\Delta R=-1$. This takes place with intensity of $\lambda_{s}$. Hence,

$$
\begin{aligned}
Q\left(\left(n_{d}+1\right)^{2},\left(n_{d}+1\right)^{2}-1\right) & =\lambda_{s} \\
Q\left(\left(n_{d}+1\right)^{2},\left(n_{d}+1\right)^{2}\right) & =-\lambda_{s}
\end{aligned}
$$

Also when the market is in $\omega\left(n_{d},-n_{d}\right)$, the only possible change is change of $\Delta R=+1 . \omega\left(n_{d},-n_{d}\right)$ is located at $Q\left(\left(n_{d}+1\right)^{2}, n_{d}^{2}+1\right)$. Hence,

$$
\begin{aligned}
& Q\left(\left(n_{d}+1\right)^{2}, n_{d}^{2}+1\right)=\lambda_{d} \\
& Q\left(\left(n_{d}+1\right)^{2}, n_{d}^{2}+2\right)=-\lambda_{d}
\end{aligned}
$$




\begin{tabular}{|c|c|c|c|}
\hline & \multicolumn{2}{|c|}{$R$} \\
\hline & & even & odd \\
\hline \multirow[t]{2}{*}{$N_{1}$} & even & $\begin{array}{c}\omega(n, r) \\
\omega(n,-r+2)\end{array}$ & $\begin{array}{c}\omega(n, r) \\
\omega(n,-r)\end{array}$ \\
\hline & odd & $\begin{array}{c}\omega(n, r) \\
\omega(n,-r)\end{array}$ & $\begin{array}{c}\omega(n, r) \\
\omega(n,-r+2)\end{array}$ \\
\hline
\end{tabular}

Table 9: Pairs of Inventory States with Symmetric Densities Their densities of $X$ are line symmetric. The line of symmetry is $x=0.5$. For given $n$, value of $r$ is such that $-n<r$

For cases of $0 \leq N_{1} \leq n_{d}$ and $R= \pm n$, there are only two cases of transition. For example, $\omega(n, n)$ has varDelta $N_{1}=+1$ or varDelta $R=-1 . \omega(n, n)$ is located $Q\left((n+1)^{2},(n+1)^{2}\right) \cdot \omega(n+1, n)$ is located $Q\left((n+2)^{2},(n+2)^{2}-1\right)$.

$$
\begin{aligned}
Q\left((n+1)^{2},(n+1)^{2}\right) & =\lambda_{s} \\
Q\left((n+1)^{2},(n+1)^{2}-1\right) & =\lambda_{s} \\
Q\left((n+2)^{2},(n+2)^{2}-1\right) & =n \theta_{1}
\end{aligned}
$$

\section{B Derivation of Heterogeneity Effect}

We need to calculate, for a given $m, n$ and $r$,

$$
\operatorname{Pr}\left(\Delta N_{1}=m-n\right) \sum_{l \neq-m}(\mu(m, l)-\mu(n, r))^{2} \operatorname{Pr}(\Delta R=l-r)
$$

where $-n_{d}<m, n<n_{d}$ We use normal distribution to approximate

$\operatorname{Pr}(\Delta R=l-r)$. For every $m$, equation (56) increases as $\alpha$ increases. It was not necessary to $\operatorname{Pr}\left(\Delta N_{1}=m-n\right)$. The first term of equation (45) increases, as $\alpha$ increases.

As for evaluating the second term, we use a pair of the symmetric $\mu(n, r)$ 's. As $\alpha$ increases, $\mu(n, r)^{\prime}$ s of equation (40) diverge from 0.5 over on $S$ interval. The symmetric pairs remain so. One of the following holds for applicable cases as listed in Table (9).

$$
\begin{aligned}
& \mu(n, r)+\mu(n,-r)=1 \\
& \mu(n, r)+\mu(n,-r+2)=1
\end{aligned}
$$

Also by assumption (a1), $\operatorname{Pr}(\Delta R=l-r)=\operatorname{Pr}(\Delta R=-l+r)$. Among the symmetric pair of $\mu(m, l)$ and $\mu(m,-l)$,

$$
\begin{aligned}
& (\mu(m, l)-\mu(n, r)) \operatorname{Pr}(\Delta R=l-r) \\
& \quad=-(\mu(m,-l)-\mu(n,-r)) \operatorname{Pr}(\Delta R=-l+r)
\end{aligned}
$$


The pair of $\mu(m, l)$ and $\mu(m,-l)$ in the above share the same probability of $\Delta N_{1}$; $\operatorname{Pr}\left(\Delta N_{1}=m-n\right)$. The weighted values of $\mu(m, l)-\mu(n, r)$ and $\mu(m,-l)-\mu(n,-r)$ cancel out in the second term equation (45). While $\alpha$ increases, the second term of equation (45) remains unchanged. Thus, as reservation prices becomes more heterogeneous, variance of bid rate increases.

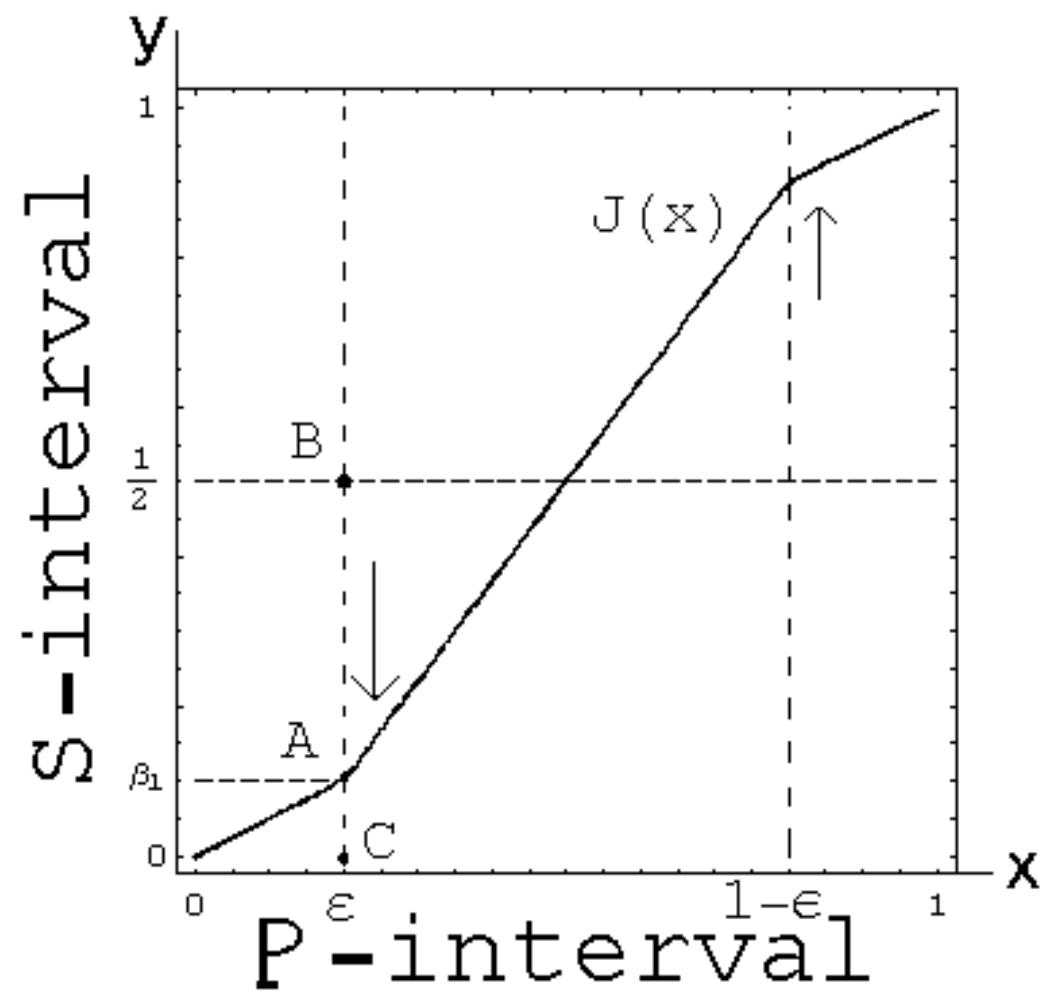

Figure 1: Function $J(x)$ For a given $\epsilon$ as defined in (a3) as the example, it must hold that $\alpha>\frac{\epsilon}{\beta_{1}}$. And $\beta_{1}<0.5$. If $\alpha \approx \frac{\epsilon}{\beta_{1}}$, the point A, where $J(x)$ has a kink, is located near point $\mathrm{B}$ on $x=\epsilon$ line. As $\alpha$ increases, point A moves toward point $\mathrm{C}$. If $\alpha=1$, then $J(x)$ is 45 degree line. 